\title{
Security for Distributed Deep Neural Networks Towards Data Confidentiality \& Intellectual Property Protection
}

\author{
Laurent Gomez ${ }^{1}$, Marcus Wilhelm ${ }^{2}$, José Márquez ${ }^{3}$ and Patrick Duverger ${ }^{4}$ \\ ${ }^{1}$ SAP Security Research, SAP Global Security \\ ${ }^{2}$ Hasso Plattner Institute, University of Potsdam \\ ${ }^{3}$ SAP Portfolio Strategy \& Technology Adoption, SAP SE \\ ${ }^{4}$ Logistic \& IT Services, City of Antibes Juan-les-Pins \\ \{laurent.gomez\}@sap.com, \{marcus.wilhelm\}@student.hpi.com, \{jose.marquez\}@sap.com, \\ \{patrick.duverger\}@antibes.com
}

\begin{abstract}
Keywords: Distributed Systems, Neural Networks, Intellectual Property, Data Protection \& Privacy, Fully Homomorphic Encryption.

Abstract: $\quad$ Current developments in Enterprise Systems observe a paradigm shift, moving the needle from the backend to the edge sectors of those; by distributing data, decentralizing applications and integrating novel components seamlessly to the central systems. Distributively deployed AI capabilities will thrust this transition.

Several non-functional requirements arise along with these developments, security being at the center of the discussions. Bearing those requirements in mind, hereby we propose an approach to holistically protect distributed Deep Neural Network (DNN) based/enhanced software assets, i.e. confidentiality of their input \& output data streams as well as safeguarding their Intellectual Property.

Making use of Fully Homomorphic Encryption (FHE), our approach enables the protection of Distributed Neural Networks, while processing encrypted data. On that respect we evaluate the feasibility of this solution on a Convolutional Neuronal Network (CNN) for image classification deployed on distributed infrastructures.
\end{abstract}

\section{Introduction}

\subsection{Motivation}

Until now, the backend (on-prem \& cloud) deployments were considered as the single source of truth \& unique point of access in regards of Enterprise Systems (ES). Nevertheless, a paradigm shift has been recently observed, by the deployment of ES assets towards the Edge sectors of the landscapes; by distributing data, decentralizing applications, de-abstracting technology and integrating edge components seamlessly to the central backend systems.

Capitalizing on recent advances on High Performance Computing along with the rising amounts of publicly available labeled data, Deep Neural Networks (DNN), as an implementation of AI, have and will revolutionize virtually every current application domain as well as enable novel ones like those on autonomous, predictive, resilient, self-managed, adaptive, and evolving applications.

\subsection{Problem Statement}

Independant Software Vendors aim to protect both: data and the Intellectual Property of their AI-based software assets, deployed on potentially unsecure edge hardware \& platforms [Goodfellow, 2018].

The deployment of data processing capabilities throughout Distributed Enterprise Systems rises several security challenges related to the protection of input \& output data [Parliament and Council, 2016] as well as of software assets.

In the specific context of distributed intelligence, DNN based/enhanced software will represent key investments in infrastructure, skills and governance, as well as in the acquisition of data and talents. The software industry is therefore in the direct need to safeguard these strategic investments by enforcing the protection of this new form of Intellectual Property.

\subsection{State-of-the-Art}

Security of Deep Neural Networks is a current research topic taking advantage of two major crypto- 
graphic approaches: variants of Fully Homomorphic Encryption/FHE [Gentry, 2009] and Secure MultiParty Computation/SMC [Cramer et al., 2015]. While FHE techniques allow addition and multiplication on encrypted data, SMC enables arithmetic operations on data shared across multi-parties.

Several approaches can be found in the literature, at different phases of the development and deployment of DNNs.

Secure Training Secure DNN training has been addressed using FHE [Graepel et al., 2012] and SMC [Shokri and Shmatikov, 2015], disregarding protection once the trained model is to be productively deployed. Other Machine Learning models such as linear and logistic regressions have also been trained in a secure way in [Mohassel and Zhang, 2017]. In those approaches, confidentiality of training data is guaranteed, while runtime protection (i.e. input, model, output) is out of scope.

Processing on Encrypted Data At processing phase, SMC has led to cooperative solutions where several devices work together to obtain federated inferences [Liu et al., 2017], not supporting deployment of the trained DNN to trusted decentralized systems. DNN processing on FHE encrypted data is covered in CryptoNets [Gilad-Bachrach et al., 2016] More recently, in [Boemer et al., 2018], the authors proposed a privacy-preserving framework for deep learning, making use of the SEAL [SEAL, 2018] FHE library. While disclosure of data at runtime is prevented in these solutions, protection of DNN models remains out of the scope.

Intellectual Property Protection of DNN Model In [Uchida et al., 2017], the authors tackles IP protection of DNN models through model watermarking. While infringement can be detected with this method, it can not be prevented. Furthermore, runtime protection of input, model and output are out of scope.

To the best of our knowledge, no other publication has holistically tackled the protection of both trained DNN models and data, targeting distributed untrusted systems.

\subsection{Data \& Intellectual Property Protection for Deep Neural Networks}

In this paper we propose a novel approach for the Intellectual Property Protection of DNNbased/enhanced software while enabling data protec- tion at processing time, making use of concepts such as Fully Homomorphic Encryption (FHE).

Once trained, DNN model parameters (i.e. weights, biases) are encrypted homomorphically. The resulting (encrypted) DNN can be distributed across untrusted landscapes, preserving its IP while mitigating the risk of reverse engineering. At runtime, FHEencrypted insights from encrypted input data are produced by the homomorphically encrypted DNN. Confidentiality of both trained DNN, input and output data will be therefore guaranteed.

In this paper, we evaluate the overall performance (e.g. CPU, memory, disk usage) along with the accuracy of encrypted DNNs.

This paper is organized as follows: Section 2 details the fundamentals of our approach. Section 3 provides an overview of our solution. In Sections 3.3 and 4, we present the architecture and evaluation, concluding with an outlook in Section 5

\section{Fundamentals}

\subsection{Deep Neural Network}

DNNs are composed of $L$ transformation layers:

1. An input layer, the tensor of input data $\mathbf{X}$

2. $L-1$ hidden layers, mathematical computations transforming $\mathbf{X}$ sequentially.

3. An output layer, the tensor of output data $\mathbf{Y}$.

We denote the output of layer $i$ as a tensor $\mathbf{A}^{[\mathbf{i}]}$, with $\mathbf{A}^{[\mathbf{0}]}=X$, and $\mathbf{A}^{[\mathbf{L}]}=Y$. Tensors can have different sizes and number of dimensions.

Each layer $\mathbf{A}^{[\mathbf{i}]}$ depends on the mathematical computations performed at the previous layer $\mathbf{A}^{[\mathbf{i}-\mathbf{1}]}$. At each layer $\mathbf{A}^{[\mathbf{i}]}$, two types of function can be computed:

- Linear: involving polynomial operations.

- Non-linear, involving non-linear operations, so called activation function, such as max, exp, division, ReLU, or Sigmoid.

\subsubsection{Linear Computation Layer}

For the sake of clarity, we exemplify the inner linear computation with a Fully Connected (FC) layer, as depicted in Figure 1 .

A Fully Connected layer, noted $\mathbf{A}^{[\mathbf{i}]}$, is composed of $n$ parallel neurons, performing a $\mathbb{R}^{n} \rightarrow \mathbb{R}^{n}$ transformation (see Figure 1). We define:

$\mathbf{a}^{[\mathbf{i}]}=\left[a_{0}^{[i]} \ldots a_{k}^{[i]} \ldots a_{N}^{[i]}\right]^{T}$ as the output of layer $\mathrm{A}^{[i]}$; 


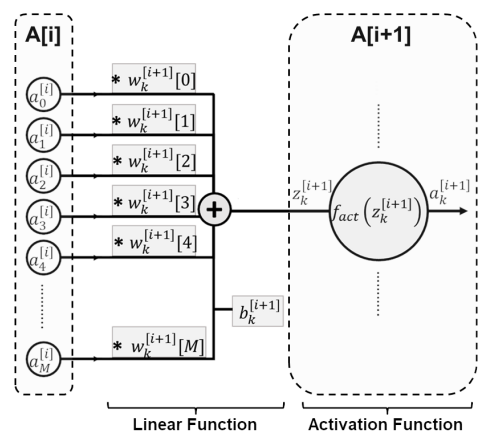

Figure 1: Fully Connected layer with Activation Function

$\mathbf{z}^{[\mathrm{i}]}=\left[z_{0}^{[i]} \ldots z_{k}^{[i]} \ldots z_{N}^{[i]}\right]^{T}$ as the linear output of layer $\mathrm{A}^{[i]} ;\left(\mathbf{z}^{[\mathbf{i}]}=\mathbf{a}^{[\mathbf{i}]}\right.$ if there is no activation function) $\mathbf{b}^{[\mathbf{i}]}=\left[b_{0}^{[i]} \ldots b_{k}^{[i]} \ldots b_{N}^{[i]}\right]^{T}$ as the bias for layer $\mathrm{A}^{[i]}$; $\mathbf{W}^{[\mathbf{i}]}=\left[\mathbf{w}_{\mathbf{0}}^{[\mathbf{i}]} \ldots \mathbf{w}_{\mathbf{k}}^{[\mathbf{i}]} \ldots \mathbf{w}_{\mathbf{N}}^{[\mathbf{i}]}\right]^{T}$ as the weights for layer $\mathrm{A}^{[i]}$.

Neuron $k$ performs a linear combination of the output of the previous layer $\mathbf{a}^{[\mathbf{i}-\mathbf{1}]}$ multiplied by the weight vector $\mathbf{w}_{\mathbf{k}}^{[\mathbf{i}]}$ and shifted with a bias scalar $b_{k}^{[i]}$, obtaining the linear combination $z_{k}^{[i]}$ :

$$
z_{k}^{[i]}=\left(\sum_{l=0}^{M} w_{k}^{[i]}[l] * a_{l}^{[i-1]}\right)+b_{k}^{[i]}=\mathbf{w}_{\mathbf{k}}^{[\mathbf{i}]} * \mathbf{a}^{[\mathbf{i}-\mathbf{1}]}+b_{k}^{[i]}
$$

Vectorizing the operations for all the neurons in layer $A^{[i]}$ we obtain the dense layer transformation:

$$
\mathbf{z}^{[\mathbf{i}]}=\mathbf{W}^{[\mathbf{i}]} * \mathbf{a}^{[\mathbf{i}-\mathbf{1}]}+\mathbf{b}^{[\mathbf{i}]}
$$

where $\mathbf{W}$ and $\mathbf{b}$ are the parameters for layer $A^{[i]}$.

\subsubsection{Activation Functions}

Activation functions are the major source of nonlinearity in DNNs. They are performed element-wise $\left(\mathbb{R}^{0} \rightarrow \mathbb{R}^{0}\right.$, thus easily vectorized), and are generally located after linear transformations such as Fully Connected layers.

$$
a_{k}^{[i]}=f_{a c t}\left(z_{k}^{[i]}\right)
$$

Several activation functions have been proposed in the literature but Rectified Linear Unit (ReLU) is currently considered as the most efficient activation function for DL. Several variants of ReLU exist, such as Leaky ReLU [Maas et al., 2013], ELU [Clevert et al., 2015] or its differentiable version Softplus.

$$
\begin{aligned}
\operatorname{ReLU}(z) & =z^{+}=\max (0, z) \\
\operatorname{Softplus}(z) & =\log \left(e^{z}+1\right)
\end{aligned}
$$

\subsection{Homomorphic Encryption}

While preserving data privacy, Homomorphic Encryption (HE) schemes allow certain computations on ciphertext without revealing neither its inputs nor its internal states. Gentry [Gentry, 2009] first proposed a Fully Homomorphic Encryption (FHE) scheme, which theoretically could compute any kind of arithmetic circuit, but is computationally intractable in practice. FHE evolved into more efficient schemes preserving addition and multiplication over encrypted data, such as BGV [Brakerski et al., 2011] , FV [Fan and Vercauteren, 2012] or CKKS [Cheon et al., 2018], allowing approximations of multiplicative inverse, exponential and logistic function, or discrete Fourier transformation. Similar to asymmetric encryption, a public-private key pair (pub, priv) is generated.

Definition 1. An encryption scheme is called homomorphic over an operation $\odot$ if it supports the following

$$
\begin{gathered}
E n c_{\text {pub }}(m)=\langle m\rangle_{\text {pub }}, \forall m \in \mathcal{M} \\
\left\langle m_{1} \odot m_{2}\right\rangle_{\text {pub }}=\left\langle m_{1}\right\rangle_{\text {pub }} \odot\left\langle m_{2}\right\rangle_{\text {pub }}, \forall m_{1}, m_{2} \in \mathcal{M}
\end{gathered}
$$

where $E n c_{\text {pub }}$ is the encryption algorithm and $\mathcal{M}$ is the set of all possible messages.

Definition 2. Decryption is performed as follows

$$
\begin{gathered}
E n c_{\text {pub }}(m)=\langle m\rangle_{\text {pub }}, \forall m \in \mathcal{M} \\
\operatorname{Dec}_{\text {priv }}\left(\langle m\rangle_{\text {pub }}\right)=m
\end{gathered}
$$

where Dec priv is the decryption algorithm and $\mathcal{M}$ is the set of all possible messages.

\subsection{Challenges}

Even though HE schemes seem theoretically promising, their usage comes with several drawbacks, particularly when applied to Deep Learning.

\subsubsection{Noise budget}

In Gentry's lattice-based HE schemes [Gentry, 2009] and subsequent variants of it, ciphertexts contain a small term of random noise drawn from some probability distribution. To estimate the current magnitude of noise, a noise budget can be calculated, that starts as a positive integer, decreases with subsequent operations and reaches 0 exactly when the ciphertext becomes indecipherable. The noise budget is more strongly affected by multiplications as by additions.

In order to cope with that challenge, encryption parameters can be adjusted accordingly to the required computation depth of an arithmetic circuit. 


\subsubsection{FHE libraries and APIs}

Multiple FHE libraries are available [Halevi and Shoup, 2014], [PALISADE, 2018], [SEAL, 2018], [Ducas and Micciancio, 2015]. Depending on the supported HE schemes, those libraries show noticeable difference on performance (e.g. computational, memory consumption), on supported operations type (e.g. addition, multiplication, negative, square, division), datatype (e.g. floating point, integer), and chipset infrastructure (e.g. CPU, GPU).

In addition, and regardless on their level of maturity and performance, HE libraries can be configured through several encryption parameters such as:

- Polynomial degree or modulus: which determines the available noise budget and strongly affects the performance.

- Plaintext modulus: which is mostly associated to the size of input data.

- Security parameter: which sets the reached level of security in bits of the cryptosystem (e.g. 128, 192, 256-bit security level).

Fine-tuning of those encryption parameters enables developers to optimize the performance of encryption and encrypted operations. The selection of the right encryption parameters depends on the size of the plaintext data, targeted accuracy loss or level of security.

\subsubsection{Linear function support only}

By construction, linear functions, composed of addition and multiplication operations, are seamlessly protected by FHE. But, non-linear activation functions such as ReLU or Sigmoid require approximation to be computed with FHE schemes.

The challenge lies on the transformation of activation functions into polynomial approximations supported by HE schemes. We elaborate more on approximation of activation functions in Section 3.2.

\subsubsection{Supported plaintext type}

The vast majority of HE schemes allow operations on integers [Halevi and Shoup, 2014 SEAL, 2018], while others use booleans [Chillotti et al., 2018] or floating point numbers [Cheon et al., 2018, SEAL, 2018]. In the case of integer supporting HE schemes, rational numbers can be approximated using fixedpoint arithmetic by scaling with a scaling factor and rounding.

\subsubsection{Performance}

FHE schemes are computationally expensive and memory consuming. In addition, ciphertexts are often significantly bigger than plaintexts and thus use more memory and disk space.

Even if in the past years the performance of FHE made it impractical, recent FHE schemes show promising throughput. New FHE libraries take also advantage of GPU acceleration.

In addition, modern implementations of $\mathrm{HE}$ schemes such as HELib [Halevi and Shoup, 2014], SEAL [SEAL, 2018], or PALISADE [PALISADE, 2018] benefit from Single Instruction Multiple Data (SIMD), allowing multiple integers to be stored in a single ciphertext and vectorizing operations, which can accelerate certain applications significantly.

\section{Approach}

As introduced in Section 1.2, the delivery of DNN-enriched insights come at a cost. ISVs aim to guarantee data security, together with the IP protection of their DNN-based software assets, deployed on potentially unsecure edge hardware \& platforms. In order to achieve those security objectives on DNN, we utilize FHE schemes to operate on ciphertext at runtime.

Consequently, secure training of DNN is out of scope of our approach as we focus on runtime execution. We assume that DNN training already preserves both data privacy and confidentiality, and the resulting trained model. Once a model is trained, as discussed in Section 2.1.1, we obtain a set of parameters for each DNN layer; i.e weights $\mathbf{W}^{[\mathbf{i}]}$ and biases $\mathbf{b}^{[i]}$. Those parameters constitute the IP to be protected when deploying a DNN to distributed systems.

\subsection{Linear Computation Layer Protection}

Our approach is agnostic from the type of layer. In [Gomez et al., 2018], we detail the encryption of layers such as Convolutional Layer or Batch Normalization. For sake of simplicity, we exemplify the encryption of DNN layers parameters on FC layers. Since FC are simply a linear transformation on the previous layer's outputs, encryption is achieved straight- 
forwardly as follows

$$
\begin{aligned}
\left\langle\mathbf{z}^{[\mathrm{i}]}\right\rangle_{\text {pub }} & =\left\langle\mathbf{W}^{[\mathbf{i}]} * \mathbf{a}^{[\mathbf{i}-\mathbf{1}]}+\mathbf{b}^{[\mathrm{i}]}\right\rangle_{\text {pub }} \\
& =\left\langle\mathbf{W}^{[\mathbf{i}]}\right\rangle_{\text {pub }} *\left\langle\mathbf{a}^{[\mathbf{i}-\mathbf{1}]}\right\rangle_{\text {pub }}+\left\langle\mathbf{b}^{[\mathrm{i}]}\right\rangle_{\text {pub }}
\end{aligned}
$$

\subsection{Activation Function Protection}

Due to their innate non-linearity, activation functions need to be approximated with polynomials to be encrypted with FHE. Several approaches have been elaborated in the literature. In [Livni et al., 2014] and [Gilad-Bachrach et al., 2016], the authors proposed to use a square function as activation function. The last layer, a sigmoid activation function, is only applied during training. Chabanne et al. used Taylor polynomials around $x=0$, studying performance based on the polynomial degree [Chabanne et al., 2017]. In [Hesamifard et al., 2017], Hesamifard et al. approximate instead the derivative of the function and then integrate to obtain their approximation.

Regardless on the approximation technique, we denote activation function $f_{\text {act }}()$ approximation as

$$
\mathbf{f}_{\text {act }}() \approx \mathbf{f}_{\text {approxact }}()
$$

By construction, we have

$$
\begin{aligned}
\left\langle\mathbf{a}_{\mathbf{k}}^{[i]}\right\rangle_{\text {pub }} & =\left\langle\mathbf{f}_{\text {act }}\left(\mathbf{z}_{\mathbf{k}}^{[i]}\right)\right\rangle_{\text {pub }} \\
& \equiv\left\langle\mathbf{f}_{\text {approxact }}\left(\mathbf{z}_{\mathbf{k}}^{[\mathbf{i}]]}\right)\right\rangle_{\text {pub }}
\end{aligned}
$$

\subsection{Architecture}

In this section we outline the architecture of our IP protection system, as depicted in Figure 2

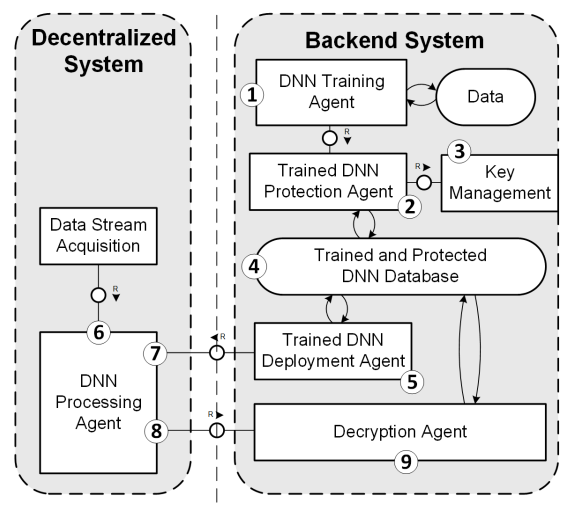

Figure 2: Overall Architecture

\subsubsection{Encryption of trained DNN}

In the backend, a DNN is trained within a DNN Training Agent, (1). The outcome of the training (NN architecture and parameters) is pushed to the Trained DNN Protection Agent, (2). Alternatively, an already trained DNN can be imported directly into the Protection Agent. The DNN Protection Agent generates a Fully Homomorphic key pair from the Key Generator component, (3). The DNN is then encrypted and stored together with its homomorphic key pair in the Trained and Protected DNN Database, (4).

\subsubsection{Deployment of trained and protected DNN}

At the deployment phase, the Trained DNN Deployment Agent deploys the DNN on distributed systems, together with its public key, (5).

\subsubsection{DNN processing}

On the distributed system, data is collected by a Data Stream Acquisition component, (6), and forwarded to the DNN Processing Agent, (7). The input layer does not involve any computation, and therefore can be seamlessly FHE encrypted as follows:

$$
\mathbf{X} \stackrel{\text { encryption }}{\longrightarrow} E n c_{\text {pub }}(X)=\langle X\rangle_{\text {pub }}
$$

Encrypted inferences are sent to the Decryption Agent, (8), for their decryption using the private key associated to the DNN, (9). FHE encryption propagates across the DNN layers, from the input to the output layer. By construction, the output layer is therefore encrypted homomorphically.

The decryption of the last layer's output $\mathbf{Y}$ is done with the private key priv:

$$
\left\langle\mathbf{A}^{[\mathbf{L}]}\right\rangle_{\text {pub }} \stackrel{\text { decryption }}{\longrightarrow} \operatorname{Dec}_{\text {priv }}\left(\left\langle\mathbf{A}^{[\mathbf{L}]}\right\rangle_{\text {pub }}\right)=\mathbf{Y}
$$

The Intellectual Property of the DNN, together with the input \& output results, is protected from any disclosure on the distributed system throughout the entire process.

\section{Evaluation}

As detailed in Section 2.3, FHE introduces additional computational costs at each step of the DNN life-cycle. In this section, we evaluate performance overhead from computation time, memory load and disk usage perspectives at DNN model and processing encryption and output decryption. 


\subsection{Hardware Setup}

As backend, we use a NVIDIA DGX-1 1 server, empowered with 8 Tesla V100 GPUs. This machine is theoretically not resource-constrained (computation $\&$ memory). We reasonably neglect the impact of the performance overhead introduced by FHE on DNN trained model encryption and output decryption.

We deploy and execute our encrypted DNN on a NVIDIA Jetson-TX2 2 , Powered by NVIDIA Pascal architecture, this platform embeds 256 CUDA cores, CPU HMP Dual Denver 22 MB L2 + Quad ARM ${ }^{\circledR}$ A572 MB L2, and 8 GB of memory. This platform gets closer to the hardware configuration of a Distributed Enterprise System.

\subsection{Software Setup}

DNN Model As demonstrated in Section 3, our approach is fully agnostic from NN topology, or implementation. For the sake of our evaluation, involving several modifications to the $\mathrm{NN}$ model, we choose a simple CNN classifier ${ }^{3}$ implemented with the Keras library ${ }^{4}$ Two datasets have been used in our experiment: CIFAR10 ${ }^{5}$ for image classification, and MNIST $^{6}$ for handwritten digits classification.

As depicted in Figure 3, we distinguish two main parts in this CNN: a feature extractor and a classifier. The feature extractor reduces the amount of information from the input image, into a set of high level and more manageable features. This step facilitates the subsequent classification of the input data.

Composed of four layers, $[F C \rightarrow \operatorname{ReLU} \rightarrow F C \rightarrow$ Softmax], the classifier categorizes the input data according to the extracted features, and outputs discrete probability distribution over 10 classes of objects.

As reference point, we evaluate key performance figures at model training and processing time without encryption. Once trained, the size of the CNN plaintext model is $9.6 \mathrm{Mb}$. On Jetson TX2, single unencrypted image classification is computed on average in $89.1 \mathrm{~ms}$.

Performances vary from 2.1s for an encrypted classification, with only $53.9 \mathrm{Mb}$ consumed memory, up to $1 \mathrm{~h} 33 \mathrm{~m}$ with almost $5 \mathrm{~Gb}$ of consumed memory.

\footnotetext{
${ }^{1}$ https://www.nvidia.com/en-us/data-center/dgx-1/

${ }^{2} \mathrm{https}: / /$ www.nvidia.com/en-us/autonomousmachines/embedded-systems-dev-kits-modules/

${ }^{3}$ https://github.com/keras-

team/keras/blob/master/examples/cifar10_cnn.py

${ }^{4}$ https://keras.io

${ }^{5} \mathrm{https}: / /$ www.cs.toronto.edu/ kriz/cifar.html

${ }^{6}$ http://yann.lecun.com/exdb/mnist/
}

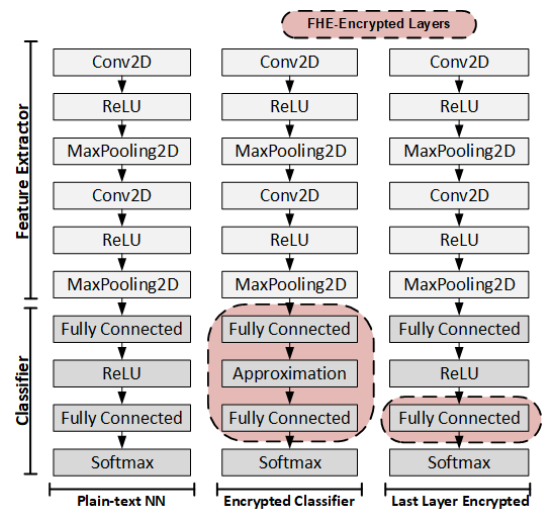

Figure 3: Keras Convolutional Neural Network.

FHE library As introduced in section 2.3, several libraries are available for FHE. We use SEAL [SEAL, 2018] C library from Microsoft Research running on CPU. This choice is motivated by the library's performance, support of multiple schemes such as BGV [Brakerski et al., 2011], stability, and documentation. The use of SEAL, implemented in $\mathrm{C}++$, with the Keras Python library requires some engineering efforts. To enable both fast performance of the native $\mathrm{C}++$ library and rapid prototyping using Python, we use Cython 7

We conduct our evaluation with the BGV scheme [Brakerski et al., 2011], utilizing the integer encoding with SIMD support. To handle the floating-point DNN parameters, we use fixed-point arithmetic with a fixed scaling factor, similarly to CryptoNets [GiladBachrach et al., 2016]. This has no noticeable impact on the classification accuracy, if a suitable scaling factor is applied. The SIMD operations allow for optimized performance through vectorization.

\subsection{Linearization}

We tackle the problem of linearization of the ReLU functions following approaches: we approximate it with a modified square function, and we skip activation function. The modified square function $x^{2}+2 x$ (see Figure 6 is derived from the ReLU approximation proposed in [Chabanne et al., 2017]. In order to optimize the computation of that function on ciphertexts, we used simpler coefficients.

In order evaluate the impact of these approaches, we trained the CNN on the CIFAR10 and MNIST datasets, replacing the last ReLU activation. Depicted in Figure 4 and Figure 5, we report the accuracy loss. Both approximations have merely a minor impact on the output classification accuracy.

\footnotetext{
${ }^{7}$ https://cython.org/
} 
Skipping the last activation function shows good results on this simple $\mathrm{CNN}$, but we do not want to generalize to any other DNN or dataset.

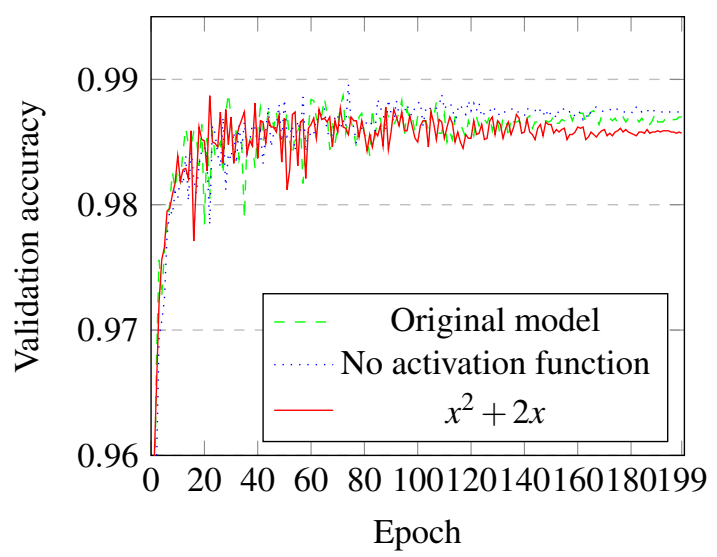

Figure 4: Classification Accuracy with ReLU Approximation - MNIST Dataset.

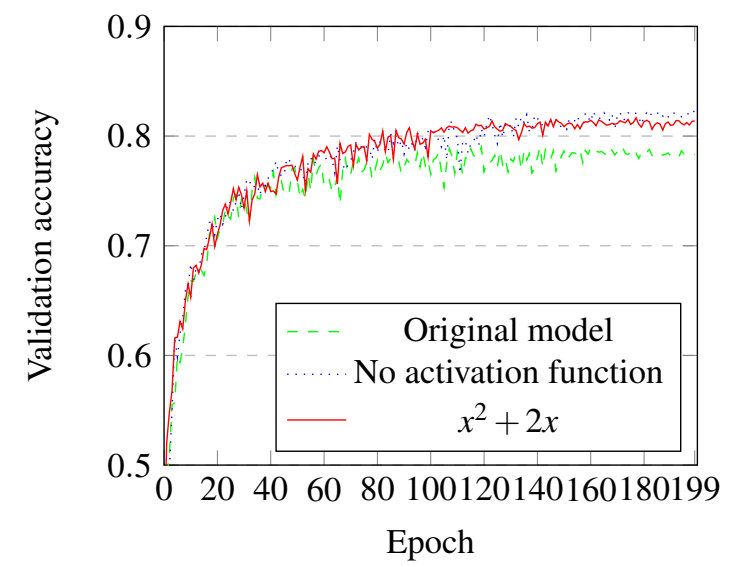

Figure 5: Classification Accuracy with ReLU Approximation - CIFAR10 Dataset.

\subsection{Experimentation Results}

\subsubsection{Model \& Data Protection}

Intellectual Property-wise, we consider the feature extractor as of minor importance, as CNNs generally use state of the art feature extractor. The IP of the model rather lies in the parameters, weights and bias, of the trained classifier. For that reason, we encrypt the classifier only, as a first step towards full model encryption, as depicted in Figure 3. To better understand the impact of computation depth, we also complete our evaluation with the encryption of the last FC layer only.

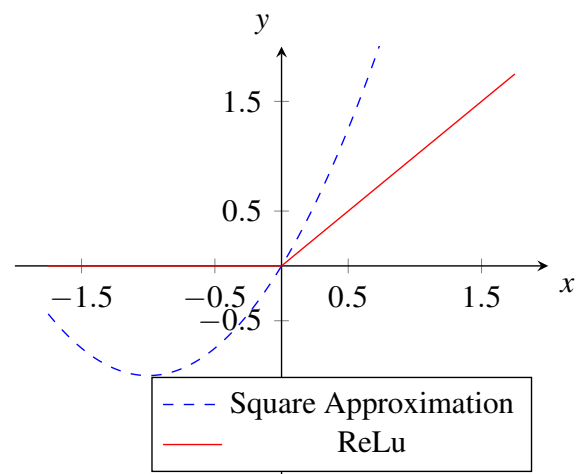

Figure 6: ReLU Approximation as Square Function

Confidentiality-wise, we evaluate the impact of extracted features encryption by comparing processing performance on an encrypted model with plaintext and encrypted feature extractor outputs.

As depicted in Figure 3, we evaluate our approach on three modified versions of the model:

- Last FC Layer Encrypted

- Full Classifier Encrypted with no Activation Function

- Full Classified Encrypted with our Modified Square Activation Function

Confidentiality-wise, we evaluate the impact of extracted features encryption by comparing processing performance on an encrypted model with plaintext and encrypted feature extractor outputs.

In order to optimize our approach, we omit the Softmax layer within the classifier. This layer does not have any influence on the classification results, as Softmax layer is mostly required at training phase, to normalize network outputs probability distribution, for more consistent loss calculations.

The overall experiment as described in section 3.3 has been applied 5 times on each model. We report average evaluation metrics for each step: model encryption, processing encryption and decryption.

\subsubsection{DNN Model Encryption}

Each trained CNN model is encrypted on DGX-1's CPU. In Table ??, we depict the resource consumption average on the following metrics:

- Time to Compute: Time to encrypt the model.

- Model Size: Size of resulting encrypted model.

- Memory Load: Overall memory usage for model encryption.

We target three security levels: 128, 192, and 256bits. For each of those, we optimize SEAL parame- 
ters as introduced in section 2.3 maximizing performance, and minimizing leftover noise budget.

Compared to the plaintext model size $(9.6 \mathrm{Mb})$, encrypted model size increases by a factor of 8,22 in the best case.

\subsubsection{DNN Processing Encryption}

The three encrypted CNN models deployed on JetsonTX2 for CPU based encrypted processing. At this stage, we evaluate the following metrics

- Time to compute: Processing time for an encrypted classification.

- Memory: Memory usage for encrypted classification.

- Remaining Noise Budget: At the end of processing encryption, we evaluate the remaining noise budget, which determines if additional encryption operations could be performed on the output vector.

In Table 1 and Table 2, we depict the performance of encrypted processing with plaintext and encrypted previous layer outputs. We study the impact of confidentality preservation of the preceding layer outputs. SEAL library supports secure computation over plaintext and ciphertext producing ciphertext. As a consequence, output of the last MaxPooling2D layer can be processed in FHE-encrypted Fully Connected layer. Secure computation between plaintext and ciphertext has a lower impact on performance.

We observe a slight performance improvement on time to compute and memory between 128 and 192bit security level. This is due to the FHE parameters optimization as described in Section 4.4.2, where initial noise budget is oversized for 128-bit security level, which has a direct impact to performance.

Experiment results show that, depending on the level of achieved security, and targeted scenario, we can achieve at best encrypted classification in $2.1 \mathrm{~s}$ (for 128 level security and only one layer encrypted).

\subsubsection{Decryption}

Following our approach, encrypted output are decrypted by the backend, on DGX-1. We therefore consider decryption as not computationally expensive, compared to encryption. Results are available in Table 3

\section{Conclusion}

In this paper, we discuss and evaluate a holistic approach for the protection of distributed Deep Neural
Network (DNN) enhanced software assets, i.e. confidentiality of their input \& output data streams as well as safeguarding their Intellectual Property. On that matter, we take advantage of Fully Homomorphic Encryption (FHE). We evaluate the feasibility of this solution on a Convolutional Neural Network (CNN) for image classification.

Our evaluation on NVIDIA DGX-1 and JetsonTX2 shows promising results on the CNN image classifier. Performances vary from $2.1 \mathrm{~s}$ for an encrypted classification, with only $53.9 \mathrm{Mb}$ consumed memory, up to $1 \mathrm{~h} 33 \mathrm{~m}$ with almost $5 \mathrm{~Gb}$ of consumed memory.

\section{REFERENCES}

Boemer, F., Ratner, E., and Lendasse, A. (2018). Parameterfree image segmentation with SLIC. Neurocomputing, 277:228-236.

Brakerski, Z., Gentry, C., and Vaikuntanathan, V. (2011). Fully homomorphic encryption without bootstrapping. Cryptology ePrint Archive, Report 2011/277.

Chabanne, H., de Wargny, A., Milgram, J., Morel, C., and Prouff, E. (2017). Privacy-preserving classification on deep neural network. IACR Cryptology ePrint Archive, 2017:35.

Cheon, J. H., Han, K., Kim, A., Kim, M., and Song, Y. (2018). Bootstrapping for approximate homomorphic encryption. IACR Cryptology ePrint Archive, 2018:153.

Chillotti, I., Gama, N., Georgieva, M., and Izabachène, M. (2018). Tfhe: Fast fully homomorphic encryption over the torus. Cryptology ePrint Archive, Report 2018/421. https://eprint.iacr.org/2018/421.

Clevert, D.-A., Unterthiner, T., and Hochreiter, S. (2015). Fast and accurate deep network learning by exponential linear units (elus). arXiv preprint arXiv:1511.07289.

Cramer, R., Damgård, I. B., et al. (2015). Secure multiparty computation. Cambridge University Press.

Ducas, L. and Micciancio, D. (2015). Fhew: bootstrapping homomorphic encryption in less than a second. In $A n$ nual International Conference on the Theory and Applications of Cryptographic Techniques, pages 617640. Springer.

Fan, J. and Vercauteren, F. (2012). Somewhat practical fully homomorphic encryption. Cryptology ePrint Archive, Report 2012/144.

Gentry, C. (2009). A Fully Homomorphic Encryption Scheme. PhD thesis, Stanford University, Stanford, CA, USA. AAI3382729.

Gilad-Bachrach, R., Dowlin, N., Laine, K., Lauter, K., Naehrig, M., and Wernsing, J. (2016). Cryptonets: Applying neural networks to encrypted data with high throughput and accuracy. In International Conference on Machine Learning, pages 201-210.

Gomez, L., Ibarrondo, A., Márquez, J., and Duverger, P. (2018). Intellectual property protection for dis- 


\begin{tabular}{|l|l|l|l|l|l|l|l|l|l|l|}
\cline { 2 - 9 } \multicolumn{1}{c|}{} & \multicolumn{9}{c|}{ Achieved Security Level (bits) } \\
\cline { 2 - 10 } \multicolumn{1}{c|}{} & \multicolumn{1}{c|}{128} & \multicolumn{1}{c|}{192} & 256 & 128 & 192 & 256 & 128 & 192 & 256 \\
\cline { 2 - 10 } \multicolumn{1}{c|}{} & \multicolumn{2}{c|}{ Full Classifier $-x^{2}+2 x$} & Full Classifier - No Act. & \multicolumn{4}{c|}{ Last Layer } \\
\hline Time to Compute (s) & 287.2 & 174.6 & 1221.3 & 43.7 & 32.9 & 90.2 & 2.1 & 2.1 & 4.5 \\
\hline Memory Load (Mb) & 4683.1 & 2924.3 & 4899.2 & 1162.5 & 869.2 & 2342.3 & 53.9 & 54.0 & 117.7 \\
\hline Remaining Noise Budget & 221.6 & 80.2 & 88.8 & 91.4 & 23.8 & 16.2 & 58.8 & 20.2 & 60.2 \\
\hline
\end{tabular}

Table 1. Runtime Encryption with Plaintext Input

\begin{tabular}{|l|l|l|l|l|l|l|l|l|l|l|}
\cline { 2 - 10 } \multicolumn{1}{c|}{} & \multicolumn{9}{c|}{ Achieved Security Level (bits) } \\
\cline { 2 - 10 } \multicolumn{1}{c|}{} & \multicolumn{1}{c|}{128} & 192 & 256 & 128 & \multicolumn{1}{c|}{192} & 256 & 128 & 192 & 256 \\
\cline { 2 - 10 } \multicolumn{1}{c|}{} & \multicolumn{2}{c|}{ Full Classifier $-x^{2}+2 x$} & Full Classifier - No Act. & \multicolumn{4}{c|}{ Last Layer } \\
\hline Time to Compute (s) & 2902.6 & 1048.6 & 5627 & 367.1 & 272.2 & 835.9 & 19.2 & 19.2 & 40.2 \\
\hline Memory Load (Mb) & 5047.8 & 5809 & 4906 & 2314.8 & 1733.7 & 4644.5 & 119.5 & 118.5 & 253 \\
\hline Remaining Noise Budget & 206.0 & 65.0 & 76.0 & 79.0 & 11.0 & 3.0 & 45.0 & 10.0 & 52.0 \\
\hline
\end{tabular}

Table 2. Runtime Encryption with Encrypted Input

\begin{tabular}{|l|l|l|l|l|l|l|l|l|l|l|}
\cline { 2 - 9 } \multicolumn{1}{c|}{} & \multicolumn{9}{c|}{ Achieved Security Level (bits) } \\
\cline { 2 - 10 } & \multicolumn{1}{c|}{128} & 192 & 256 & 128 & 192 & \multicolumn{1}{c|}{256} & 128 & 192 & 256 \\
\cline { 2 - 10 } & Full Classifier $-x^{2}+2 x$ & Full Classifier - No Act. & \multicolumn{4}{c|}{ Last Layer } \\
\hline Time to Compute (s) & 2.9 & 1.7 & 3.2 & 0.6 & 0.6 & 1.0 & 0.2 & 0.1 & 0.2 \\
\hline Memory Load (Mb) & 963.8 & 397.4 & 2062.5 & 123.4 & 73.4 & 267.1 & 17.8 & 17.8 & 38.7 \\
\hline
\end{tabular}

Table 3. Decryption - Performance

tributed neural networks - towards confidentiality of data, model, and inference. In Samarati, P. and Obaidat, M. S., editors, Proceedings of the 15th International Joint Conference on e-Business and Telecommunications, ICETE 2018 - Volume 2: SECRYPT, Porto, Portugal, July 26-28, 2018., pages 313-320. SciTePress.

Goodfellow, I. (2018). Security and privacy of machine learning. RSA Conference.

Graepel, T., Lauter, K., and Naehrig, M. (2012). Ml confidential: Machine learning on encrypted data. In International Conference on Information Security and Cryptology, pages 1-21. Springer.

Halevi, S. and Shoup, V. (2014). Algorithms in helib. In International cryptology conference, pages 554-571. Springer.

Hesamifard, E., Takabi, H., and Ghasemi, M. (2017). Cryptodl: Deep neural networks over encrypted data. CoRR, abs/1711.05189.

Liu, J., Juuti, M., Lu, Y., and Asokan, N. (2017). Oblivious neural network predictions via minionn transformations. In Proceedings of the 2017 ACM SIGSAC Conference on Computer and Communications Security, pages 619-631. ACM.

Livni, R., Shalev-Shwartz, S., and Shamir, O. (2014). On the computational efficiency of training neural networks. In Ghahramani, Z., Welling, M., Cortes, C., Lawrence, N. D., and Weinberger, K. Q., editors, Advances in Neural Information Processing Systems 27, pages 855-863. Curran Associates, Inc.

Maas, A. L., Hannun, A. Y., and Ng, A. Y. (2013). Rectifier nonlinearities improve neural network acoustic models. In Proc. icml, volume 30, page 3.
Mohassel, P. and Zhang, Y. (2017). Secureml: A system for scalable privacy-preserving machine learning. In Security and Privacy (SP), 2017 IEEE Symposium on, pages 19-38. IEEE.

PALISADE (2018). The palisade lattice cryptography library.

Parliament, E. and Council (2016). General data protection regulation.

SEAL (2018). Simple Encrypted Arithmetic Library (release 3.1.0). https://github.com/Microsoft/ SEAL Microsoft Research, Redmond, WA.

Shokri, R. and Shmatikov, V. (2015). Privacy-preserving deep learning. In Proceedings of the 22nd ACM SIGSAC conference on computer and communications security, pages 1310-1321. ACM.

Uchida, Y., Nagai, Y., Sakazawa, S., and Satoh, S. (2017). Embedding watermarks into deep neural networks. In Proceedings of the 2017 ACM on International Conference on Multimedia Retrieval, pages 269-277. ACM. 